\title{
TWO PROOFS IN COMBINATORIAL NUMBER THEORY
}

\author{
JAROSLAV NEŠTKRIL AND VOJTĚCH RÖDL
}

\begin{abstract}
The aim of this paper is to present a short combinatorial proof of a theorem of P. Erdős on multiplicative bases of integers. A solution of a problem of P. Erdős and D. J. Newman is also presented.
\end{abstract}

I. Let $X$ be a set of positive integers. We say that $X$ is a multiplicative base if for every positive integer $n$ there are $x, y \in X$ such that $n=x y$. The following was proved by P. Erdős (see [1]).

THEOREM 1. Let $X$ be a multiplicative base. Then for every positive integer $p$ there exists a positive integer $n$ such that $n$ can be expressed as the product of two elements of $X$ in at least $p$ different ways.

In the proof of Theorem 1 we shall need the well-known theorem of Ramsey: Let $p$ be a given integer and let $[A]^{p}=C_{1} \cup C_{2}$ be a partition of the set of all p-tuples of elements of an infinite set $A$ into two parts. Then there exists $i$ and an infinite set $B \subseteq A$ such that $[B]^{p} \subseteq C_{i}$. The set $B$ is called homogeneous with respect to the partition $\left(C_{1}, C_{2}\right)$.

Proof of Theorem 1. In the following we shall consider the integers which are products of distinct primes only. Such integers can be identified in a natural way with finite subsets of the set of all primes. Thus it suffices to prove the following

TheOREM $1^{\prime}$. Let $A$ be an infinite set. Denote by $[A]^{<\omega}$ the set of all finite subsets of A. Let $\mathscr{A} \subseteq[A]^{<\omega}$ be a set of finite subsets of $A$ such that the following holds:

$$
\begin{aligned}
& \text { For every } P \in[A]^{<\omega} \text { there are } Q, Q^{\prime} \in \mathscr{A} \text { such that } \\
& Q \cup Q^{\prime}=P \text { and } Q \cap Q^{\prime}=\varnothing .
\end{aligned}
$$

Then for every integer $p$ there is a set $P$ which can be expressed in at least p-different ways as a union of two disjoint elements of $\mathscr{A}$.

Proof of Theorem 1' is a straightforward consequence of Ramsey's theorem: Let $p$ be a given positive integer, $p \geqslant 2$. For every $i=1, \ldots, p-1$ consider a partition $[A]^{i}=C_{1}^{i} \cup C_{2}^{i}$ defined by $Q \in C_{1}^{i}$ iff $Q \in \mathscr{A}$. Let $B$ be an infinite set which is homogeneous with respect to all partitions $C_{1}^{i}, C_{2}^{i}, i=1, \ldots, p-1$ (such a set clearly

Received by the editors May 12, 1982 and, in revised form, January 19, 1984.

1980 Mathematics Subject Classification. Primary 05A17, 10K30.

Key words and phrases. Multiplicative base, Ramsey theorem, $B^{(k)}$-sequence. 
exists by iterating the Ramsey theorem). From $(*)$ we get that there is an $i, 1 \leqslant i \leqslant$ $p-1$, such that

$$
[B]^{i} \subseteq C_{1}^{i} \text { and }[B]^{p-i} \subseteq C_{1}^{p-i},
$$

and hence every $P \in[B]^{p}$ can be represented as a union of at least $\left(\begin{array}{l}p \\ i\end{array}\right) \geqslant p$ elements of $\mathscr{A}$.

REMARK. Note that the above proof gives nothing concerning the additive version of Theorem 1. This is an old problem of P. Erdős:

Problem. Let $X$ be a set of positive integers with the property that for every positive integer $n$ there are $x, y \in X$ such that $n=x+y$. Is is true that for every positive integer $p$ there exists a positive integer $n$ such that $n$ can be expressed as the sum of two elements of $X$ in at least $p$ different ways?

II. Let $X$ be a set of positive integers. We say that $X$ is a $B_{2}^{k}$-sequence if the number of representations of $n$ as the sum $x+y, x, y \in X$, is at most $k$ and for some $n$ is exactly $k$. The following is known as the Erdős-Newman problem:

Is it true that given a $k$ there exists a $B_{2}^{(k)}$-sequence $X$ such that for every partition $X=X_{1} \cup \cdots \cup X_{r}$ into a finite number of parts one of the parts is a $B_{2}^{(k)}$ sequence?

For certain $k$ the affirmative solution is given in [2]. We prove here (by different methods)

THEOREM 2. For every $k \geqslant 2$ there exists a set of integers $X$ such that:

(1) $X$ is a $B_{2}^{(k)}$-sequence;

(2) For every partition of $X$ into a finite number of parts one of the parts is a $B_{2}^{(k)}$-sequence.

The proof is based on the existence of Ramsey graphs of a special type. Before stating this result we introduce some necessary notions. An ordered graph is a graph $(V, E)$ with a (fixed) ordering of its vertices. $K_{3}$ is the complete graph with 3 vertices (i.e. the triangle). Denote by $L_{k}$ the graph $(V, E)$ defined as follows:

$$
\begin{aligned}
& V=\{0,1, \ldots, k+1\}, \\
& E=\{\{0, i\} ; i=1, \ldots, k\} \cup\{\{i, k+1\} ; i=1, \ldots, k\} .
\end{aligned}
$$

The graph $L_{k}$ is always considered as an ordered graph with the natural ordering of its vertices. (The graph $L_{k}$ is depicted in Figure 1.) Let $(V, E)$ and $\left(V^{\prime}, E^{\prime}\right)$ be ordered graphs. We say that $(V, E)$ is contained in $\left(V^{\prime}, E^{\prime}\right)$ if $V \subseteq V^{\prime}$ and $E \subseteq E^{\prime}$ and the ordering of $V^{\prime}$ restricted to the set $V$ coincides with that of $V$. We shall use the following

LEMMA. For every $k, r \geqslant 2$ there exists an ordered graph $G_{r}^{k}=(V, E)$ with the following properties:

(1) $G_{r}^{k}$ does not contain $L_{k+1}$ and $K_{3}$;

(2) for every partition $E=E_{1} \cup \cdots \cup E_{r}$ one of the classes $E_{i}$ contains $L_{k}$.

(A proof of this Lemma is too nontrivial (and lengthy) to be included here. However it follows easily from the "partite construction" which is introduced and studied in [3].) 


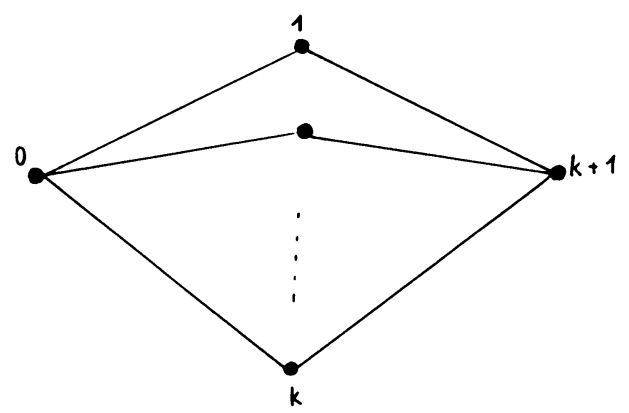

Figure 1

Proof of Theorem 2. Put $G=\bigcup\left(G_{r}^{k} ; r \geqslant 2\right)$-the disjoint union of graphs described in the Lemma. Consider $G=(V, E)$ as an ordered graph which contains every $G_{r}^{k}, r \geqslant 2$. Assume without loss of generality that the vertices of $G$ are integers. Define the set $X$ as the set of all sums $\sum 3^{n}$, where the summation is taken over the set $I_{u v}$ of all positive integers $n$ which satisfy $u \leqslant n<v$ for an edge $\{u, v\} \in E$. In the sequel the sets of the form $I_{u v}$ are called intervals. We prove that $X$ has the desired properties:

ad 1. Observe that if $z=x+y$ for $x, y \in X$ and if $z=\sum_{i \in I} \varepsilon_{i} 3^{i}$ is the triadic expansion of $z$ (i.e. $\varepsilon_{i}=1$ or 2), then $I$ is either an interval or union of two intervals. Moreover, if $\varepsilon_{i}=2$ for an $i \in I$, then $I$ is an interval and $I_{1}=\left\{i ; \varepsilon_{i}=2\right\}$ is also an interval. Therefore we may distinguish three cases which can be visualized as follows:

(i) $I_{1} \neq \varnothing$. Either:

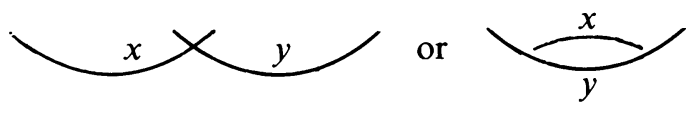

(ii) $I_{1}=\varnothing, I$ is an interval:

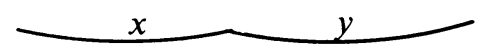

(iii) $I$ is the union of two intervals, but it is not an interval itself:

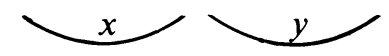

In (i) there are at most two (depicted) possibilities for $z=x^{\prime}+y^{\prime}, x^{\prime}, y^{\prime} \in X$. In (ii), there are at most $k$ possibilities for the solution $z=x^{\prime}+y^{\prime}$ by assumption (1) of the Lemma on graphs $G_{r}^{k}$ (graphs $G_{r}^{k}$ do not contain $L_{k+1}$ ). In (iii), $z=x+y$ is the unique solution. Therefore $X$ is a $B_{2}^{(k)}$-sequence.

As every partition of $X$ corresponds to a partition of the edges of the graph $G$, the second half of the statement of Theorem 2 follows immediately from property (2) of the Lemma on the graphs $G_{r}^{k}$.

The methods described above enable the following generalizations of Theorems 1 and 2. Let $X$ be a set of positive integers. Let us call $X$ the multiplicative $j$-base if for every positive integer $n$ there are $x_{1}, \ldots, x_{j} \in X$ such that $n=x_{1} \cdots x_{j}$.

THEOREM I. Let $X$ be a multiplicative $j$-base. Then for every positive integer $p$ there exists a positive integer $n$ such that $n$ can be expressed as the product of $j$ elements of $X$ in at least $p$ different ways. 


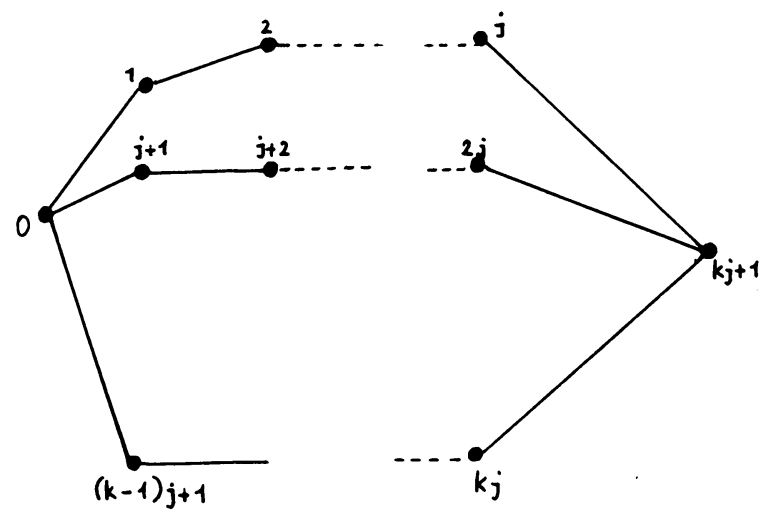

FIGURE 2

Similarly, let us say that $X$ is a $B_{j}^{(k)}$-sequence if the number of representation of $n$ as the sum $n=x_{1}+x_{2}+\cdots+x_{i}, i \leqslant j, x_{1}, x_{2}, \ldots, x_{i} \in X$, is at most $k$ and for some $n$ exactly $k$. P. Erdős asked whether an analogy of Theorem 2 can be proved for $B_{j}^{(k)}$-sequences. If we use a suitable modification of graphs $L_{k}$, Theorem 2 can be strengthened as follows:

THEOREM II. For every $k \geqslant 2$ and $j \geqslant 2$ there exists a set of integers $X$ such that

(1) $X$ is a $B_{j}^{(k)}$ sequence;

(2) for every partition of $X$ into a finite number of parts one of the parts is a $B_{j}^{(k)}$-sequence.

The proof is analogous to the above proof of Theorem 2 with the difference that instead of graphs $L_{k}$ we use graphs $L_{k}^{j}=(V, E)$ defined as follows:

$$
\begin{aligned}
V= & \{0,1, \ldots, k j+1\}, \\
E= & \{\{0, j \cdot i+1\} ; i=0, \ldots, k-1\} \\
& \cup\{\{j \cdot i+l, j i+l+1\}, i=0,1, \ldots, k-1, l=1,2, \ldots, j-1\} \\
& \cup\{\{l j, k j+1\} ; l=1, \ldots, k\}
\end{aligned}
$$

(see Figure 2).

\section{REFERENCES}

1. P. Erdős, On the multiplicative representation of integers, Israel J. Math. 2 (1964), 251-261.

2. Some application of Ramsey's theorem to additive number theory, European J. Combin. 1 (1980), 43-46.

3. J. Nešetril and V. Rödl, Simple proof of the existence of restricted Ramsey graphs by means of partite construction, Combinatorica 2 (1981), 199-202.

KKIOV MFF UK, Charles University, Malostranske Nám. 25,110 00 Praha 1, Czechoslovakia

Department of Mathematics, FJFi ČVUT, Husova 5, 11000 Praha 1, Czechoslovakia 